\title{
Realization of the first sub-shot-noise wide field microscope
}

\begin{abstract}
Nigam Samantaray ${ }^{1,2}$, Ivano Ruo-Berchera ${ }^{1}$, Alice Meda ${ }^{1}$ and Marco Genovese ${ }^{1,3}$
Recently, several proof of principle experiments have demonstrated the advantages of quantum technologies over classical schemes. The present challenge is to surpass the limits of proof of principle demonstrations to approach real applications. This letter presents such an achievement in the field of quantum enhanced imaging. In particular, we describe the realization of a sub-shot-noise wide field microscope based on spatially multi-mode non-classical photon number correlations in twin beams. The microscope produces realtime images of 8000 pixels at full resolution, for a $500 \mu \mathrm{m}^{2}$ field of view, with noise reduced to $80 \%$ of the shot noise level (for each pixel), which is suitable for absorption imaging of complex structures. By fast post-elaboration, specifically applying a quantum enhanced median filter, the noise can be further reduced (to $<30 \%$ of the shot noise level) by setting a trade-off with the resolution, thus achieving the best sensitivity per incident photon reported in absorption microscopy. Light: Science \& Applications (2017) 6, e17005; doi:10.1038/lsa.2017.5; published online 14 July 2017
\end{abstract}

Keywords: imaging; microscopy; parametric down conversion; sub-shot-noise

\section{INTRODUCTION}

Sensitivity in standard optical imaging and sensing, such as those exploiting classical illuminating fields, is fundamentally lower bounded by the shot noise, which is the inverse square root of the number of photons used. Surpassing such a limit is particularly effective when there is a constraint on the usable optical power, for example, a limit determined by the damage threshold of the sample ${ }^{1}$, the stress of the optical elements ${ }^{2}$, or alteration of chemical and biological photo sensitive process and, most fundamentally, quantum back-action ${ }^{3}$. Following the seminal works of $\mathrm{Caves}^{4}$, which have demonstrated how squeezed light can improve the sensitivity in interferometry, nonclassical states of light have long been considered to overcome shot noise, thus prompting deep theoretical investigations and many proposed schemes ${ }^{5-9}$

The experimental possibility of generating two-photon entangled states $^{10}$ (such as NOON states with $N=2$ ) and the availability of singlephoton detectors have enabled the demonstration of the potential of quantum enhanced sensing, aimed at reaching the fundamental Heisenberg limit in phase-contrast polarization microscopy ${ }^{11,12}$, magnetic field sensing ${ }^{13}$ and solution concentration measurement ${ }^{14}$.

However, almost all previous experimental results have been proof of principle demonstrations ${ }^{6}$, because of the difficulties in generating high photon number entangled states and a high photon flux (comparable to that used in classical schemes) and protecting them from decoherence up to the detection, thus limiting the optical and detection losses ${ }^{15}$.

Some remarkable results have been obtained because of recent progress in the generation of a highly non-classical single mode ${ }^{16}$ and few modes squeezed states ${ }^{17}$ : these have been successfully implemented in gravitational wave interferometry ${ }^{2}$, for particle tracking in a biological environment ${ }^{18,19}$, and to some extent, for beam displacement measurement ${ }^{20,21}$ and optical magnetometry ${ }^{22}$.

Most quantum-enhanced imaging and sensing protocols have been obtained exploiting single or few spatial modes of the quantum probe beam, such as in the case of squeezing, and with single photon detection in schemes based on two-photon correlated states. In both cases, only one parameter of the system, namely a single point of the sample, can be probed in a single run. The reconstruction of the sample as a whole requires time consuming scanning and accumulation of many detection windows. Instead, the exploitation of a high number, namely thousands, of modes in the same run is the requirement for quantum enhanced wide field imaging. In practice, the number of spatial details of a structure that can be probed at the same time is determined by the number of spatial modes enclosed in the illuminating field. Indeed, one of the challenges in quantum optics and quantum enhanced imaging is to generate and efficiently detect highly non-classical features in a multi-mode regime ${ }^{23-28}$. The first proof of principle of a quantum enhanced imaging protocol exploiting this parallelism was reported in Ref. 29, following the proposal of Ref. 30 , even if the average enhancement and the poor spatial resolution were not sufficient for any practical purposes, such as in absorption microscopy, in which the technique is naturally addressed. In this paper, we address this point by reporting the realization of a sub-shotnoise (SSN) microscope exploiting thousands of spatial modes, detected independently by the same number of pixels of a chargecoupled-device (CCD) camera operated in the linear (non-amplified)

${ }^{1}$ INRIM, Strada delle Cacce 91, I-10135 Torino, Italy; ${ }^{2}$ Politecnico di Torino, Corso Duca degli Abruzzi, 24, I-10129 Torino, Italy and ${ }^{3}$ INFN, Via P. Giuria 1, I-10125 Torino, Italy Correspondence: A Meda, Email: a.meda@inrim.it

Received 29 August 2016; revised 16 January 2017; accepted 24 January 2017; accepted article preview online 28 January 2017 
regime. Thousands of photons per pixel are detected in the exposure time of a single shot. Therefore, the microscope operates in a widefield regime (no scanning is required) and is suitable for dynamic imaging. The microscope is based on the non-classical and spatially multimode correlations of squeezed vacuum that is naturally generated by a traveling wave parametric amplifier in both low and high gain regimes ${ }^{24,26,30-32}$. The noise of the image, formed by the probe beam interacting with the sample, is locally reduced by pixel-by-pixel subtraction of the correlated noise pattern measured on the other beam $^{30}$. Moreover, we introduce the concept of a quantum enhanced median filter ${ }^{33}$ : quantum noise reduction at different spatial scales can be naturally combined with the statistical noise smoothing used in every standard image processing algorithm, thus resulting in an impressive overall enhancement in object recognition.

We attain a significant improvement in sensitivity with respect to that of any classical absorption microscopy system at the same illumination level. Our present results outperform the previous proof of principle demonstrations ${ }^{29,34}$, substantially improving the resolution by a factor of 10-100 (depending on the sensitivity level), in terms of both pixel count and the size of the imaged details of the sample.

Wide field microscopy is the simplest, fastest, least expensive and oldest imaging modality used, for example, for live cell imaging. It has the advantage of requiring the lowest photon dose, especially for absorbed light imaging. The lowest photon dose that achieves a measurable metric for the experimental question should be used, for instance to ensure that the cellular processes under investigation are not shifted to an alternate pathway due to environmental stress ${ }^{35}$. Indeed, the results presented here could potentially have immediate applications in many fields, such as biology and biochemistry. Furthermore, the comparison with the reference beam can be used to provide the absolute value of the absorption, thus providing the possibility for quantitative analysis of the related properties. With small modifications (essentially in the data processing), our technique can also serve as a basis for enhancing sensitivity in schemes with different goals: for example, ghost imaging ${ }^{36-41}$, detection and imaging in the presence of environmental or electronic noise background ${ }^{42,43}$ and accurate characterization of retina rod-cell response to single photon stimulation ${ }^{44}$. Finally, this new capability can also be used in quantum radiometry, for example, for the absolute calibration of detectors with spatial resolution, as demonstrated in Refs 45-48.

\section{MATERIALS AND METHODS}

In absorption wide-field imaging, as in standard microscopy, a probe illuminates the sample all at once and the transmitted pattern is imaged by the detector, typically as the pixel array of a camera. The intensity measured by each pixel, $N_{\alpha}$, here expressed in number of photons, has a mean expectation value $\left\langle N_{\alpha}\right\rangle=(1-\alpha)\langle N\rangle$, where $\langle N\rangle$ is the mean number of detected photons per pixel in absence of the sample and $\alpha$ is the absorption coefficient. The photon noise of the measurement can be obtained by modeling the absorption as the action of a beam splitter of transmittance $1-\alpha$ on the beam with initial variance $\left\langle\Delta^{2} N\right\rangle$ (Ref. 49), thus resulting in the form $\left\langle\Delta^{2} N_{\alpha}\right\rangle=\left[(1-\alpha)^{2}(F-1)+1-\alpha\right]\langle N\rangle$, where $F=\left\langle\Delta^{2} N\right\rangle /\langle N\rangle$ is the Fano factor in absence of the sample. The value $F=1$ establishes a bound between classical and non-classical photon statistics. In particular, $F$ is lower bounded by the unity for classical states, whereas specific non-classical states may have sub-Poissonian photon statistics, that is, $0 \leq F<1$. The uncertainty of the absorption estimation in the direct (DR) imaging scheme is therefore

$$
\Delta \alpha_{\mathrm{DR}}=\frac{\sqrt{\left\langle\Delta^{2} N_{\alpha}\right\rangle}}{\left|\partial_{\alpha}\left\langle N_{\alpha}\right\rangle\right|}=\sqrt{\frac{(1-\alpha)^{2}(F-1)+1-\alpha}{\langle N\rangle}}
$$

The limit of the sensitivity for a classical probe $(F=1)$ is $\Delta \alpha=\sqrt{(1-\alpha) /\langle N\rangle}$, representing, for small absorption, the shotnoise limit with the typical scaling of the inverse square root of the number of photons. However, by inspecting Equation (1), it is clear that non-classical optical fields with Fano factor smaller than one allow beating the shot noise limit. We note that the Fano factor, appearing in Equation (1), is usually deteriorated with respect to the value of $F_{0}$ for the unperturbed (pure) state, due to optical losses, including detector quantum efficiency. In particular, one obtains $F=\eta F_{0}+1-\eta$ where we define the overall detection probability $0 \leq \eta \leq 1$. Thus, the non-classical behavior, in terms of noise reduction, has a lower bound of $F_{\text {loss }}=1-\eta$. It must be emphasized that splitting a single mode beam into $n$ pixels leads to a detection probability of the order of $\eta \leq 1 / n$ for each pixel, ruling out the possibility of using single modes for SSN wide-field imaging for any reasonable number of pixels. Thus, the necessity of having many non-classical spatial modes is evident, each addressing a single pixel with limited losses. Even if sub-Poissonian light beams were obtained as single or few modes, it is not completely obvious how to generate a beam with a high number of sub-Poissonian modes and simultaneously detect them. On the other side, it is relatively simple to produce a pair of beams that are (individually) spatially incoherent but locally correlated at the quantum level, by means of traveling wave parametric amplifier in the spontaneous regime. Even if the fluctuations of a single spatial mode in one beam are super-Poissonian, these fluctuations are perfectly replicated in the correlated mode of the second beam because of photon number entanglement. This correlation is verified for all of the wide range of localized transverse spatial modes. The degree of correlation and its non-classical features can be quantified on the basis of the noise reduction factor (NRF) $\sigma=\left\langle\Delta^{2}\left(N_{1}-N_{2}\right)\right\rangle /\left\langle N_{1}+N_{2}\right\rangle$ (Refs 23,24,26,30-32,49-52), measured for a pair of pixels by collecting correlated spatial modes. The NRF indeed represents the equivalent of the Fano factor for bipartite state, in which the shot noise level becomes $\left\langle N_{1}+N_{2}\right\rangle=2\langle N\rangle$. While for classical beams the NRF is lower bounded by 1 , quantum correlation can lead to $0 \leq \sigma<1$. In particular, in the presence of losses, $\sigma=1-\eta$.

Whereas twin beams in a single spatial mode have been used to demonstrate SSN absorption measurements in a double-beam scheme $e^{53,54}$, parametric down-conversion (PDC) multi-mode quantum correlations can be used for wide-field SSN imaging ${ }^{30}$.

The object is placed in one beam, and the second beam is used as a reference. Note that the double-beam (or double-path) approach is commonly used in imaging and spectroscopy involving faint absorptions, because it enables the canceling out of classical (superPoissonian) noise and provides a direct estimation of the absolute transmittance (absorption) by instantaneous comparison with the unperturbed reference beam. We consider the intensity difference between two correlated pixels of the two beams, whose expectation value is $\left\langle N_{1}-N_{2, \alpha}\right\rangle=\alpha\langle N\rangle \quad$ (we assume balanced beams, $\left.\left\langle N_{1}\right\rangle=\left\langle N_{2}\right\rangle=\langle N\rangle\right)$. In this context, the noise can be expressed in terms of the noise reduction factor in absence of the sample, in the form $\left\langle\Delta^{2}\left(N_{1}-N_{2, \alpha}\right)\right\rangle=\left[\alpha^{2}(F-1)+\alpha+2 \sigma(1-\alpha)\right]\langle N\rangle$ (see Ref. 30). 
a

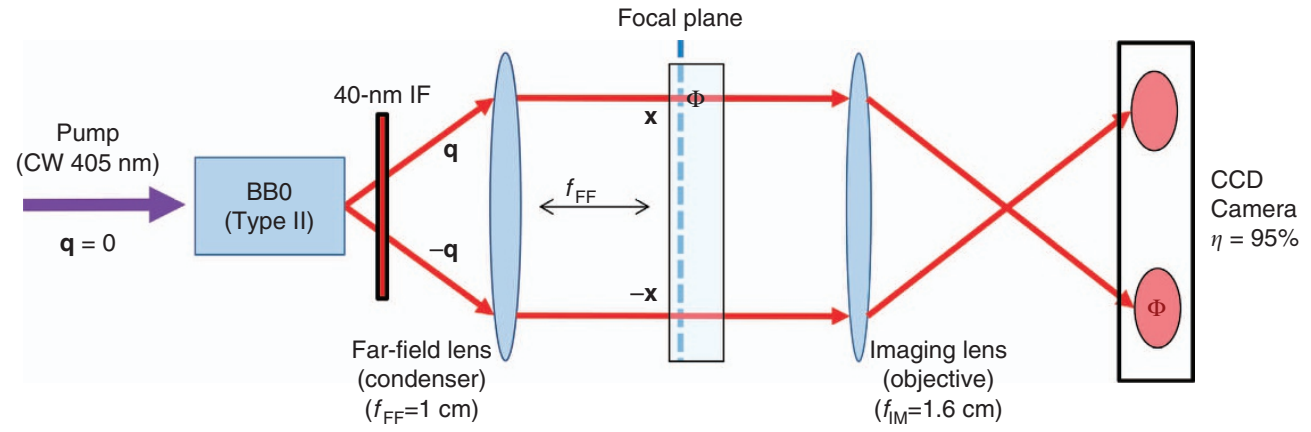

b

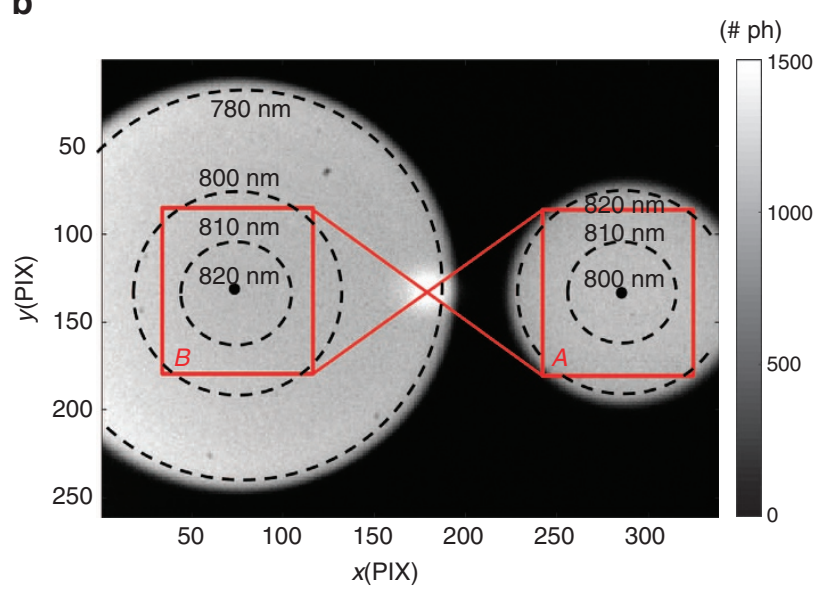

C

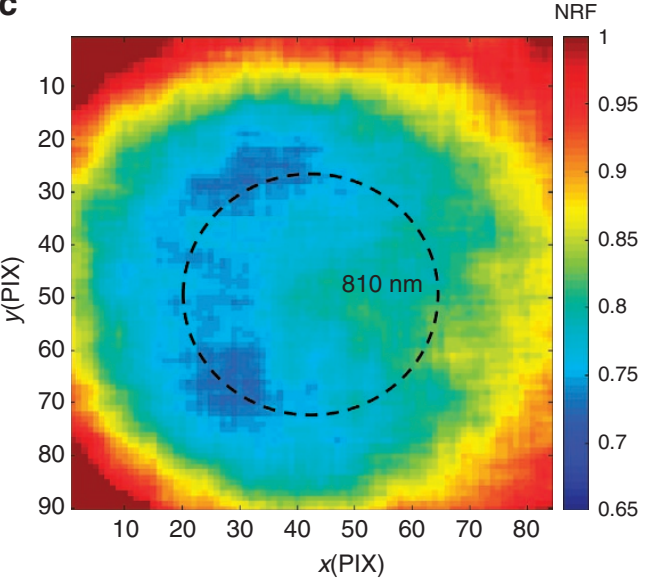

Figure 1 Experimental setup. (a) Schematic of the experiment. (b) Image of the single shot acquired by the CCD camera in $100 \mathrm{~ms}$. The pixel size is $39 \mu \mathrm{m}$, corresponding to $5-\mu \mathrm{m}$ resolution in the focal (object) plane. (c) 2D map of the NRF for $5-\mu \mathrm{m}$ resolution in the focal (object) plane.

Therefore, the absorption uncertainty in the differential (DF), both classical and quantum, imaging scheme is

$$
\Delta \alpha_{\mathrm{DF}}=\frac{\sqrt{\left\langle\Delta^{2}\left(N_{1}-N_{2, \alpha}\right)\right\rangle}}{\left|\partial_{\alpha}\left\langle N_{-, \alpha}\right\rangle\right|}=\sqrt{\frac{\alpha^{2}(F-1)+\alpha+2 \sigma(1-\alpha)}{\langle N\rangle}}
$$

The performance of the classical differential (DC) imaging scheme is derived from Equation (2) by substituting $\sigma=1$. In the situation of interest, the absorption is so small that the term $\alpha^{2}(F-1)$ is negligible even in presence of classical super-Poissonian noise of the source $(F>1)$, and the uncertainty in the differential classical imaging scheme becomes $\Delta \alpha_{\mathrm{DC}}=\sqrt{(2-\alpha) /\langle N\rangle}$, which is only a factor of $\sqrt{2}$ larger than shot-noise-limited direct imaging. Under the same condition, the quantum enhancement provided by SSN correlations with $\sigma<1$ is also quantified in terms of signal-to-noise ratio, $\mathrm{SNR}=\alpha / \Delta \alpha$, by using Equations (1) and (2), as

$$
\begin{gathered}
\frac{\Delta \alpha_{\mathrm{SSN}}}{\Delta \alpha_{\mathrm{DC}}}=\frac{\mathrm{SNR}_{\mathrm{DC}}}{\mathrm{SNR}_{\mathrm{SSN}}}=\sqrt{\frac{\alpha+2 \sigma(1-\alpha)}{2-\alpha}} \approx \sqrt{\sigma} \\
\frac{\Delta \alpha_{\mathrm{SSN}}}{\Delta \alpha_{\mathrm{DR}}}=\frac{\mathrm{SNR}_{\mathrm{DR}}}{\mathrm{SNR}_{\mathrm{SSN}}}=\sqrt{\frac{\alpha+2 \sigma(1-\alpha)}{1-\alpha}} \approx \sqrt{2 \sigma}
\end{gathered}
$$

The advantage with respect to the differential classical scheme appears when $\sigma<1$, wheras a more strict condition, $\sigma<1 / 2$, is needed to beat the direct (shot-noise-limited) scheme.

The setup for the SSN microscope scheme is sketched in Figure 1a. A CW laser beam $\left(100 \mathrm{~mW}\right.$ at $\left.\lambda_{p}=405 \mathrm{~nm}\right)$ pumps a $1-\mathrm{cm}$ type-II beta-barium-borate nonlinear crystal, in which two correlated beams are generated. The far field of the emission, where spatial correlation occurs, is realized at the focal plane of a lens with $f_{\mathrm{FF}}=1 \mathrm{~cm}$ focal length. Then, the far field plane is imaged (magnification factor $M=7.8$ ) to the detection plane by means of a second lens system with $f_{\mathrm{IM}}=1.6 \mathrm{~cm}$. The detector is a CCD camera Princeton Inst. Pixis 400BR Excelon, operating in linear mode (no electromultiplication gain), with high quantum efficiency ( $>95 \%$ at $810 \mathrm{~nm}), 100 \%$ fill factor and low noise [read noise is few $e^{-} /$(pix - frame)]. The size of the physical pixels of the camera is $13 \mu \mathrm{m}$, but here we group them by a $3 \times 3$ hardware binning. Hereafter, if not explicitly indicated, the single elementary pixel is intended to have a linear size of $39 \mu \mathrm{m}$, thus causing a sufficient reduction of the effect of the read noise for our purposes. A spectral selection is performed with two identical interferential filters $(800 \pm 20 \mathrm{~nm}$, with transmission of $99 \%)$, one just after the crystal and the other mounted on the camera. A test sample, with absorption $\alpha=1 \%$ representing the Greek letter $\Phi$ (size $300 \times 400 \mu \mathrm{m}$ ) is realized by a few nanometers thick titanium deposition on a coated glass slide.

When inserted, the letter intercepts one beam at the focal plane of the far field lens whereas the slide extends to the second beam (in the far field, the centers of the two beams are separated by $1.0 \mathrm{~mm}$ ). It is important to note that the acquisition time of a single shot is typically $10^{11}$ times the coherence time of the PDC process (on the order of $\left.10^{-12} \mathrm{~s}\right)$. Since the number of photons per pixel is $\sim 10^{3}$, the occupation of the single temporal mode is $10^{-8}$ photons/mode. Therefore, the statistics of the noise should be Poissonian, that is, shot-noise limited.

In the crystal, a photon of the pump beam is converted into a pair of photons with lower frequency, thus fulfilling the energy and momentum conservation (phase matching-condition). In particular, 
if the pump is approximated by a plane wave with transverse momentum $\mathbf{q}=0$, the two photons of a pair must fulfill $\mathbf{q}_{1}+\mathbf{q}_{2}=0$, thus being emitted with opposite transverse momenta. The lens maps momentum $\mathbf{q}$ at wavelength $\lambda$ into point $\mathbf{x}=\lambda \mathrm{fq} / 2 \pi$ at the focal plane, thus leading degenerate photons (having $\lambda_{1}=\lambda_{2}=\lambda_{d}=2 \lambda_{p}=810 \mathrm{~nm}$ ) being found in symmetric positions $\mathbf{x}_{1}=-\mathbf{x}_{2}$ with respect to the pump direction, as shown in Figure 1a. Two symmetric pixels of the camera should always detect the same number of photons. Moreover, even if the emission is broadband both in frequency and momentum, phase matching conditions establish a relationship between the wavelength and the photon direction, specifically the modulus of the transverse momentum, so that photons are emitted in concentric cones, each corresponding to a certain wavelength. In Type-II PDC, the correlated photons have orthogonal polarizations, and the emission cones have different centers. The intensity distribution, detected in a $100-\mathrm{ms}$ shot, is shown in Figure 1b. The dotted circumferences approximately represent the wavelength distribution in the two orthogonally polarized beams. By selecting two symmetric regions, $A$ and $B$, around degeneracy (represented by red squares in the picture), one expects each pixel of $A$ to be non-classically correlated with a corresponding symmetric pixel in $B$. In practice, there are two important limiting factors to this imaging system that must be considered. One limits the field of view, and the other lower bounds the spatial scale at which the noise subtraction can be efficiently performed:

- The trade-off between field of view and NRF: Near the degeneracy wavelength for correlated photons, $\lambda_{1}=\lambda_{d}+\Delta \lambda$ and $\lambda_{2}=\lambda_{d}-\Delta \lambda$; the point-to-point correlation in the far field becomes $\mathbf{x}_{1}+\mathbf{x}_{2} \cong \frac{2 \Delta \lambda}{\lambda} \mathbf{x}_{1}$. Therefore, the center of symmetry of the correlations is $\mathbf{x}=0$ only at the degenerate wavelength. As long as one moves from the degeneracy, the center of symmetry shifts proportionally to $\frac{\Delta \lambda}{\lambda_{d}}$. After the pixel grid has been positioned to be symmetric with respect to $\mathbf{x}=0$, only a relatively small spectral bandwidth around degeneracy can be tolerated (in our case, $\sim 40 \mathrm{~nm}$ ). This reflects on the available angular bandwidth, see Figure $1 \mathrm{~b}$, which corresponds to an approximately $500 \times 500 \mu \mathrm{m}$ field of view in the focal plane. Figure 1c shows a map of the NRF obtained by subtracting locally, pixel-by-pixel, the two regions $A$ and $B$. The best NRF occurs along the degenerate ring, and far from it, the correlation slightly decreases because the corresponding pixels of the two regions no longer perfectly intercept correlated directions. Moreover, right-hand part shows a further increase of the NRF, which our experience suggests can be ascribed to a small aberration in the optical system, and which we are able to decrease but not completely suppress. This technical issue can be solved by a careful analysis and realization of an ad-hoc optical system.

- The trade-off between spatial resolution and NRF: In practice, rather than a plane wave, the pump is a Gaussian beam propagating along the $z$ direction, with a waist $w_{p}$, and thus with a transverse momentum distribution centered at $\mathbf{q}=0$ with bandwidth $\Delta q \sim 2 / w_{p}$. Transverse momentum conservation $\mathbf{q}_{1}+\mathbf{q}_{2}=0 \pm \Delta \mathbf{q}$ leads to a less strict position correlation in the far field, $\mathbf{x}_{1}+\mathbf{x}_{2}= \pm \Delta \mathbf{x}$, where the relative uncertainty of the photon position is $\Delta \mathrm{x} \equiv 2 r \sim \lambda \mathrm{f} / \pi w_{p}$. A measurement of this spatial uncertainty is provided in Figure 2, representing the spatial cross correlation function of the noise patterns of two symmetric regions of $40 \times 40$ physical pixels. The cross section is Gaussian with two slightly different FWHM in the horizontal and vertical axes, estimated to be $2 r_{y}=5.6(0.2) \mu \mathrm{m}$ and $2 r_{x}=6.8(0.2) \mu \mathrm{m}$, respectively. It is clear that two symmetric pixels detect most of the correlated photons only if their size $L$ is larger than this uncertainty (see Ref. 32).

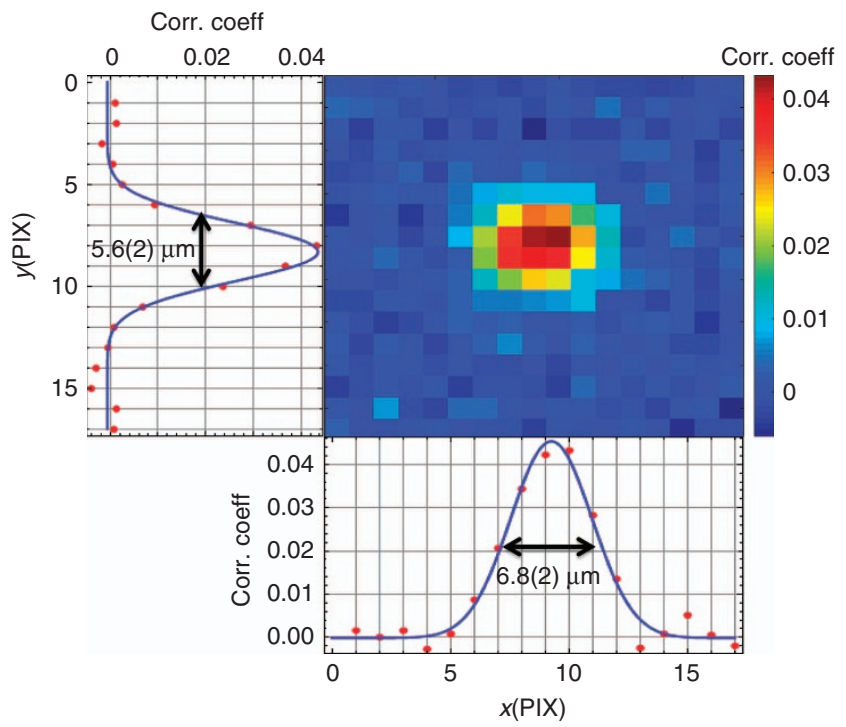

Figure 2 Spatial cross correlation function. The 2D map represents the value of the correlation coefficient between two regions of $40 \times 40$ pixels, chosen approximately in symmetric positions at the detection plane. Here, we exploited the full resolution of the camera (physical pixel of $13 \mu \mathrm{m}$ ), which corresponds to $1.7 \mu \mathrm{m}$ in the object plane. The peak represents the position in which the regions are well correlated, pixel-by-pixel. Shifting one of the regions in the pixel grid more than the spatial jitter of the correlated photon makes the correlation coefficient drop to zero. The vertical and horizontal sections are shown in the left-hand-side and bottom graphs, respectively, with their fitting Gaussian functions and the indication of the FWHMs. Corr. coeff, correlation coefficient.

\section{RESULTS AND DISCUSSION}

The NRF can be described by the function $\sigma=1-\eta_{0} \cdot \eta_{\text {coll }}$, in which the detection probability $\eta$ is split into the term $\eta_{0}$, representing the transmission-detection efficiency of the optical path, and $0<\eta_{\text {coll }}<1$, representing the collection efficiency of correlated photons. The efficiency $\eta_{\text {coll }}$ is a monotonically increasing function of the ratio $L / 2 r$, which reaches the asymptotic value $\eta_{\text {coll }} \sim 1$ for $L \gg 2 r$ (Supplementary Information). Thus, at different resolution scales, given by the size of pixels or, more generally, by the spatial scale in which the signal is integrated, the noise reduction factor and thus the SNR, according to Equation (3a) and (3b), are different. The spatial information at different scales can be recovered starting from a high resolution image in which the pixels size $x_{\text {pix }}$ can be smaller than the correlation area and by averaging the signal in groups of $d \times d$ pixels so that $L=d \cdot x_{\text {pix }}$. This is particularly useful, given that it can be interpreted as a quantum-enhanced version of the standard median filter used for noise correction in classical imaging. In the classical median filter, the value of a pixel is replaced with the mean value of its $d \times d$ neighborhood, thus enabling a reduction of the noise simply by a statistical cancelation. Of course, this comes at the expense of filter details smoothing. If the median filter is applied to the noisesubtracted image, the two effects, namely the statistical smoothing and the photon noise subtraction, combine, thereby allowing a net improvement of faint object recognition. Figure 3 shows an example of the application of the median filter to a direct image, a classical differential image, and a SSN image. The upper-right panel represents the shape of the object obtained by averaging over 300 shots at full resolution $(L=5 \mu \mathrm{m})$. The image represents the absorption coefficient, assuming values around zero outside the $\Phi$ and value around $\alpha=0.01$ inside the $\Phi$. The other panels present single shot images at different resolution scales. The SSN images are obtained by the subtraction of 


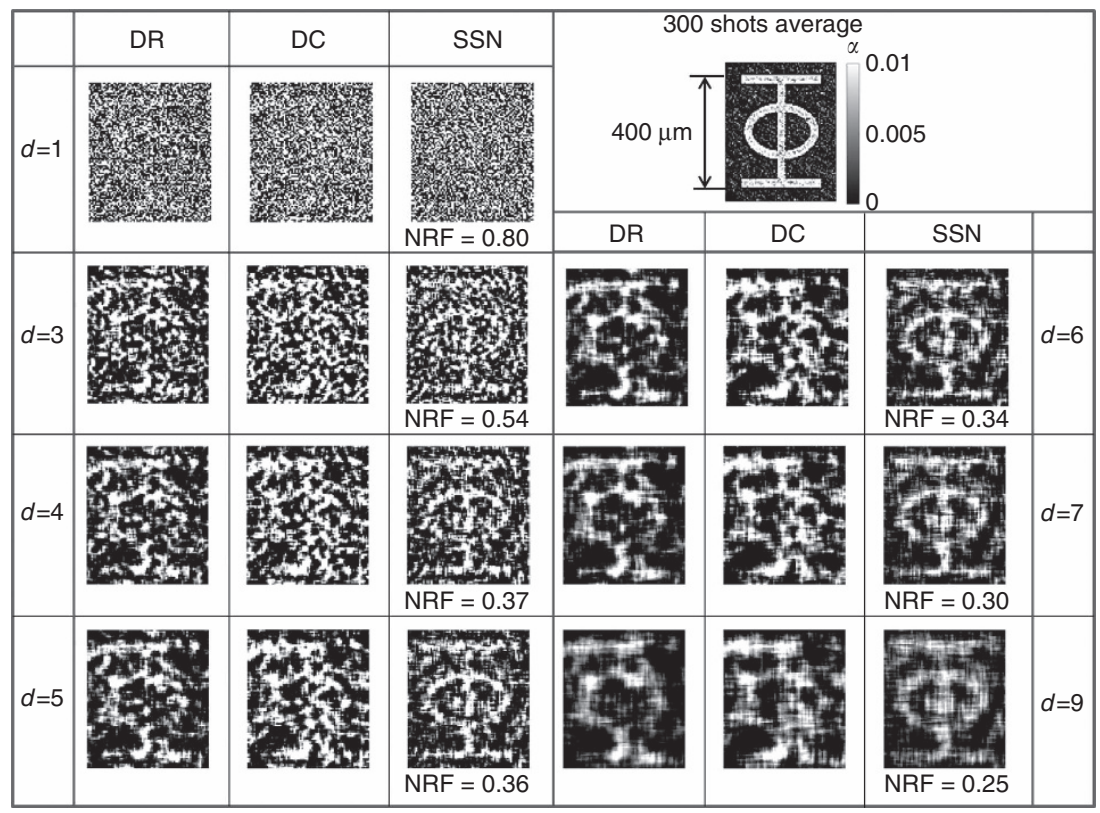

Figure 3 Application of a median filter to a single shot image for different integration scales $d$. The direct (DR) image, the differential classical (DC) image, and the SSN image are compared in each panel for the same value of $d$. Upper-left panel is the image of the object after averaging over 300 shots.

the correlated noise from the direct image. The DC images are simulated by subtracting an uncorrelated (but shot-noise-limited) noise pattern, for example, a noise region shifted more than $2 r$ from the correlated position. Notably, the single shot image of the sample is completely hidden by the shot noise at the full resolution, $d=1$. When the median filters are applied, $d>1$, the shape of the sample clearly emerges, especially for the SSN case. As discussed before, the advantage of the SSN image increases with the scale $d$ of the median filter. The DC image is the poorest because it contains twice the shot noise. Notably, DC imaging is advantageous compared with the DR imaging if classical super-Poissonian noise is present, in particular when $F>2$.

The exposure time of the single shot in our setup is $100 \mathrm{~ms}$, and the read-out time of the pixel matrix is few hundreds of milliseconds. In a few seconds, it is possible to realize a SSN movie (See Supplementary Movie) in which the sample is simply translated by a micrometer stage during the acquisition. Even in this case, the edges of the moving object are easier to follow in the SSN imaging box (right-hand side window) than in the DC imaging (central window) or in the DR imaging box (left-hand side window), thus demonstrating that our technique is suitable for dynamic imaging. With a more powerful or pulsed pump laser and the faster operating modes achievable by commercial cameras, rate of hundreds of frame/s should be reasonably attained.

The statistical analysis of the SNR is performed by acquiring $\mathcal{N}=300$ shots with and without the sample. The shots without the sample are used to estimate the NRF and Fano factor at different scales and to eliminate static intensity and efficiency gradients by a standard flat field algorithm. Each shot with the sample contains a DR shot-noise limited image and the correlated noise. The NRF of the $n$th shot is evaluated experimentally through spatial statistics over the ensemble of the correlated pixel pairs belonging to regions $A$ and $B$, namely $\sigma(n)=\frac{V_{x}\left[N_{A}^{(n)}(\mathbf{x})-N_{B}^{(n)}(-\mathbf{x})\right]}{E_{\mathrm{x}}\left[N_{A}^{(n)}+N_{B}^{(n)}\right]}$, where $E_{\mathrm{x}}[N(\mathbf{x})]=(1 / \mathscr{M}) \sum_{\mathrm{x}} N(\mathbf{x})$ is the mean value of the $\mathscr{M}$ pixel of the region and
$V_{\mathrm{x}}[N(\mathbf{x})]=E_{\mathrm{X}}\left[N^{2}(\mathbf{x})\right]-E_{\mathrm{x}}[N(\mathbf{x})]^{2}$ is the variance. The average $\mathrm{NRF}$ over the 300 values is reported in the graph in Figure 4 for different resolution scales in the object plane (see also Supplementary Information). In particular, we note that already for a resolution of $5 \mu \mathrm{m}$, comparable with the correlation FWHM of the spatial correlation function, the system reaches an NRF of $\sigma=0.8$, thus providing better performance than DC imaging (see Equation (3a)) in a matrix of $>8000$ pixels. For a resolution of $15 \mu \mathrm{m}$ the NRF is below 0.5 , which is the threshold to surpass the performance of the DR, SSN limited, imaging (see Equation (3b)). Similarly, the Fano factor is evaluated and reported in Figure 4, thus demonstrating the Poissonian character of the light statistics.

The SNR is estimated over a stripe of pixels of the images after the application of the median filter (in particular, we consider a vertical stripe inside the main axis of the letter $\Phi)$. We label $\alpha^{(n)}(\mathbf{x})$ as the absorption value of the pixel in position $\mathbf{x}$ of the $n$th shot. First, the SNR for each position is evaluated as $\operatorname{SNR}(\mathbf{x})=E_{n}[\alpha(\mathbf{x})] / V_{n}[\alpha(\mathbf{x})]^{1 / 2}$, where $E_{n}[\alpha(\mathbf{x})]=(1 / \mathcal{N}) \sum_{n=1}^{\mathcal{N}} \alpha^{(n)}(\mathbf{x})$ is the experimental temporal average of the absorption and $V_{n}[\alpha(\mathbf{x})]$ is the variance. Then, the spatial average of the $\operatorname{SNR}(\mathbf{x})$ for $\mathbf{x}$ belonging to the vertical stripe is evaluated. The experimental results, demonstrating the advantages of the quantum noise subtraction, are reported in Figure 4. The data are compared with the theoretical predictions obtained by substituting the estimated NRF in the theoretical expression of Equation (3a) and (3b). The SNR improvement of the SSN imaging is slightly higher than expected, with respect to both DR and DC imaging, because the SNR is evaluated on a vertical stripe close to the degenerate wavelength region, where the NRF is slightly lower than the average (see Figure $1 \mathrm{c}$ and the related discussion).

\section{CONCLUSIONS}

In summary, we realized the first sub-shot-noise wide field microscope, demonstrating a noise reduction of $20 \%$ below the shot noise for each resolution cell (pixel) of $5 \mu \mathrm{m}$ in a matrix of $\sim 8000$ pixels. This value is sufficient for wide-field imaging of 


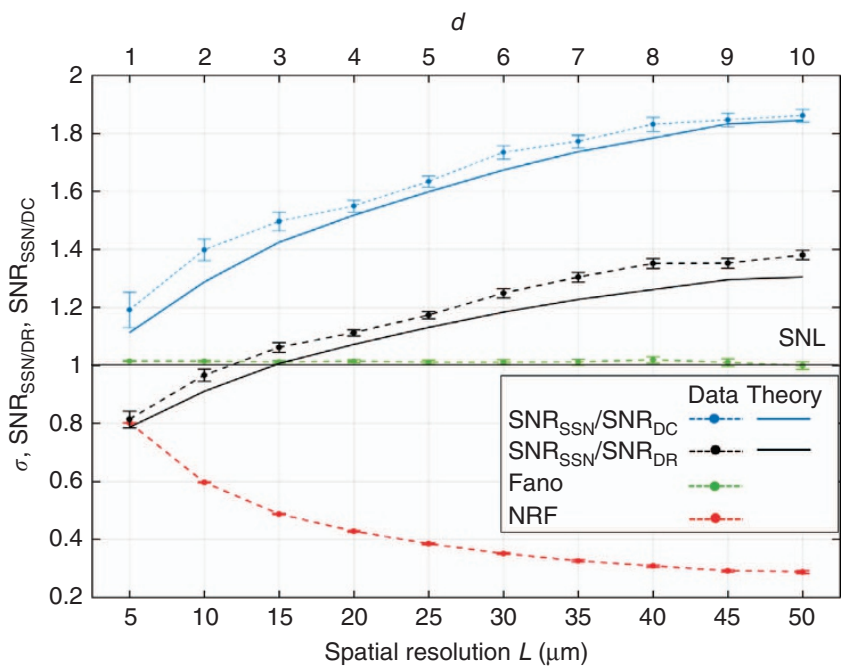

Figure 4 Experimental noise reduction factor (NRF) and signal-to-noise ratio (SNR) as a function of the resolution in the focal (object) plane $L$ (or equivalently as a function of the binning $d$ of the median filter, upper scale). Red dots represent the average of the NRF in a region of the same size as the $\Phi$ object, that is $400 \times 300 \mu \mathrm{m}$. The black dots are the SNR of the SSN images normalized to that of the direct images. For $L \geq 15 \mu \mathrm{m}$, the quantum protocol has advantages. Analogously, the blue series demonstrates that the advantage of the SSN imaging with respect to the differential classical imaging is present at any spatial resolution and reaches values of more than $80 \%$. Solid lines correspond to the quantum enhancement predicted by Equation (3a) and (3b), when the estimated values of the NRF are considered.

complex structures. Increasing the spatial scale of the details, the noise reduction factor improves accordingly; for example, a resolution of $25 \mu \mathrm{m}$ is $62 \%$ below shot noise and for $50 \mu \mathrm{m}$ is $72 \%$ on average below shot noise level. At this last scale, for example, the SNR is almost twice that of the classical differential technique for the same illumination level, whereas the sensitivity improvement with respect to classical direct imaging (obtained by a single, shot noise limited beam) is $\sim 30 \%$. Equivalently it allows one to maintain the same SNR reducing almost four times the illumination level compared with the DC imaging and almost twice compared with DR imaging. In general, to have the SNR of DC (or of DR) imaging, the photon number of the quantum beam can be decreased by a factor $\sigma$ ( $2 \sigma$ for DR). We showed that the decrease in the quantum noise at different scales is fully compatible with, and actually improves, the standard noise reduction techniques, on the basis of a posteriori elaborations of the image taken at full resolution. We demonstrated this important point with a sample made of a ultra-thin metallic depositions on a glass slide. These performance levels represent a breakthrough, filling the gap between the proof-of-principle of quantum enhanced scheme and a system suitable for applications ${ }^{6,55}$.

We believe that our technique has the potential for wide-spread use in absorption microscopy. The spatial resolution can be further improved (nothing prevents reaching the Abbe limit), and the range of applicability can be extended by engineering highly non-classical bright squeezed vacuum sources in a pulsed regime ${ }^{52}$.

\section{CONFLICT OF INTEREST}

The authors declare no conflict of interest.

\section{AUTHOR CONTRIBUTIONS}

IRB, AM and MG conceived the idea of the experiment, which was discussed and designed with input from all authors. NS, IRB, AM realized the experimental setup and collected the data in INRIM quantum optics labs (coordinated by MG). All authors discussed the results and contributed to the writing of the paper.

\section{ACKNOWLEDGEMENTS}

This work was supported by the MIUR Project 'Premiale P5'. We thank Matteo Fretto for the micro-fabrication of the object used in the measurement, F Scafirimuto for the help in a preliminary characterization of the system, and IP Degiovanni for fruitful discussions and advises.

1 Taylor MA, Bowen WP. Quantum metrology and its application in biology. Phys Rep 2016; 615: 1-59.

2 The LIGO Scientific Collaboration. A gravitational wave observatory operating beyond the quantum shot-noise limit. Nat Phys 2011; 7: 962-965.

3 Giovannetti V, Lloyd S, Maccone L. Quantum-enhanced measurements: beating the standard quantum limit. Science 2004; 306: 1330-1336.

4 Caves CM. Quantum-mechanical noise in an interferometer. Phys Rev D 1981; 23: $1693-1708$.

5 Demkowicz-Dobrzański R, Jarzyna M, Kołodyński J. Quantum limits in optical interferometry. Prog Opt 2015; 60: 345-435.

6 Genovese M. Real applications of quantum imaging. J Opt 2016; 18: 073002.

7 Kolobov MI. Quantum Imaging. New York: Springer; 2007.

8 Nabors CD, Shelby RM. Two-color squeezing and sub-shot-noise signal recovery in doubly resonant optical parametric oscillators. Phys Rev A 1990; 42: 556-559.

9 Souto Ribeiro PH, Schwob C, Maître A, Fabre C. Sub-shot-noise high-sensitivity spectroscopy with optical parametric oscillator twin beams. Opt Lett 1997; 22: 1893-1895.

10 Matthews CFJ, Alberto P, Bonneau D, O'Brien JL. Heralding two-photon and fourphoton path entanglement on a chip. Phys Rev Lett 2011; 107: 163602.

11 Ono T, Okamoto R, Takeuchi S. An entanglement-enhanced microscope. Nat Commun 2013; 4: 2426.

12 Israel Y, Rosen S, Silberberg Y. Supersensitive polarization microscopy using NOON states of light. Phys Rev Lett 2014; 112: 103604.

13 Wolfgramm F, Vitelli C, Beduini FA, Godbout N, Mitchell MW. Entanglement-enhanced probing of a delicate material system. Nat Photonics 2013; 7: 28-32.

14 Crespi A, Lobino M, Matthews JCF, Politi A, Neal CR et al. Measuring protein concentration with entangled photons. Appl Phys Lett 2012; 100: 233704.

15 Demkowicz-Dobrzański R, Kołodyński J, Guță M. The elusive Heisenberg limit in quantum-enhanced metrology. Nat Commun 2012; 3: 1063.

16 Mehmet M, Ast S, Eberle T, Steinlechner S, Vahlbruch $\mathrm{H}$ et al. Squeezed light at $1550 \mathrm{~nm}$ with a quantum noise reduction of $12.3 \mathrm{~dB}$. Opt Express 2011; 19: 25763-25772.

17 Armstrong S, Morizur JF, Janousek J, Hage B, Treps N et al. Programmable multimode quantum networks. Nat Commun 2012; 3: 1026.

18 Taylor MA, Janousek J, Daria V, Knittel J, Hage B et al. Biological measurement beyond the quantum limit. Nat Photonics 2013; 7: 229-233.

19 Taylor MA, Janousek J, Daria V, Knittel J, Hage B et al. Subdiffraction-limited quantum imaging within a living cell. Phys Rev X 2014; 4: 011017.

20 Treps N, Grosse N, Bowen WP, Fabre C, Bachor HA et al. A quantum laser pointer. Science 2003; 301: 940-943.

21 Pooser RC, Lawrie B. Ultrasensitive measurement of microcantilever displacement below the shot-noise limit. Optica 2015; 2: 393-399.

22 Wolfgramm F, Cerè A, Beduini FA, Predojevic A, Koschorreck M et al. Squeezed-light optical magnetometry. Phys Rev Lett 2010; 105: 053601.

23 Brida G, Caspani L, Gatti A, Genovese M, Meda A et al. Measurement of sub-shotnoise spatial correlations without background subtraction. Phys Rev Lett 2009; 102: 213602.

24 Jedrkiewicz O, Jiang YK, Brambilla E, Gatti A, Bache M et al. Detection of sub-shotnoise spatial correlation in high-gain parametric down conversion. Phys Rev Lett 2004; 93: 243601.

25 Mosset A, Devaux F, Lantz E. Spatially noiseless optical amplification of images. Phys Rev Lett 2005; 94: 223603

26 Blanchet JL, Devaux F, Furfaro L, Lantz E. Measurement of sub-shot-noise correlations of spatial fluctuations in the photon-counting regime. Phys Rev Lett 2008; 101: 233604.

27 Corzo NV, Marino AM, Jones KM, Lett PD. Noiseless optical amplifier operating on hundreds of spatial modes. Phys Rev Lett 2012; 109: 043602.

28 Boyer V, Marino AM, Pooser RC, Lett PD. Entangled images from four-wave mixing. Science 2008; 321: 544-547.

29 Brida G, Genovese M, Ruo-Berchera I. Experimental realization of sub-shot-noise quantum imaging. Nat Photonics 2010; 4: 227-230.

30 Brambilla E, Caspani L, Jedrkiewicz O, Lugiato LA, Gatti A. High-sensitivity imaging with multi-mode twin beams. Phys Rev A 2008; 77: 053807. 
31 Brambilla E, Gatti A, Bache M, Lugiato LA. Simultaneous near-field and far-field spatial quantum correlations in the high-gain regime of parametric down-conversion. Phys Rev A 2004; 69: 023802.

32 Agafonov IN, Chekhova MV, Leuchs G. Two-color bright squeezed vacuum. Phys Rev A 2010; 82: 011801.

33 Davies E. Computer and Machine Vision: Theory, Algorithms, Practicalities. 4th edn. Waltham: Academic Press; 2012.

34 Brida G, Genovese M, Meda A, Ruo-Berchera I. Experimental quantum imaging exploiting multimode spatial correlation of twin beams. Phys Rev A 2011; 83: 033811.

35 Cole R. Live cell imaging: the cell's perspective. Cell Adh Migr 2015; 8: 452-459.

36 Pittman TB, Shih YH, Strekalov DV, Sergienko AV. Optical imaging by means of twophoton quantum entanglement. Phys Rev A 1995; 52: R3429-R3432.

37 Meyers R, Deacon KS, Shih Y. Ghost-imaging experiment by measuring reflected photons. Phys Rev A 2008; 77: 041801(R).

38 Ferri F, Magatti D, Gatti A, Bache M, Brambilla E et al. High-resolution ghost image and ghost diffraction experiments with thermal light. Phys Rev Lett 2010; 94: 183602.

39 Brida G, Chekhova MV, Fornaro GA, Genovese M, Lopaeva ED et al. Systematic analysis of signal-to-noise ratio in bipartite ghost imaging with classical and quantum light. Phys Rev A 2011; 83: 063807.

40 Gatti A, Brambilla E, Bache M, Lugiato LA. Ghost imaging with thermal light: comparing entanglement and classical correlation. Phys Rev Lett 2004; 93: 093602.

41 O'Sullivan MN, Chan KW, Boyd RW. Comparison of the signal-to-noise characteristics of quantum versus thermal ghost imaging. Phys Rev A 2010; 82: 053803.

42 Morris PA, Aspden RS, Bell JEC, Boyd RW, Padgett MJ. Imaging with a small number of photons. Nat Commun 2015; 6: 5913.

43 Lopaeva ED, Ruo-Berchera I, Degiovanni IP, Olivares S, Brida G et al. Experimental realization of quantum illumination. Phys Rev Lett 2013; 110: 153603.

44 Phan NM, Cheng MF, Bessarab DA, Krivitsky LA. Interaction of fixed number of photons with retinal rod cells. Phys Rev Lett 2014; 112: 213601.

45 Avella A, Ruo-Berchera I, Degiovanni IP, Brida G, Genovese M. Absolute calibration of an EMCCD camera by quantum correlation, linking photon counting to the analog regime. Opt Lett 2016; 41: 1841-1844.

46 Meda A, Ruo-Berchera I, Degiovanni IP, Brida G, Rastello ML et al. Absolute calibration of a charge-coupled device camera with twin beams. Appl Phys Lett 2014, 105: 101113
47 Peřina J, Haderka O, Allevi A, Bondani M. Absolute calibration of photon-numberresolving detectors with an analog output using twin beams. Appl Phys Lett 2014; 104: 041113.

48 Agafonov IN, Chekhova MV, Iskhakov TS, Penin AN, Rytikov GO et al. Absolute calibration of photodetectors: photocurrent multiplication versus photocurrent subtraction. Opt Lett 2011; 36: 1329-1331.

49 Bondani M, Allevi A, Zambra G, Paris MGA, Andreoni A. Sub-shot-noise photon-number correlation in a mesoscopic twin beam of light. Phys Rev A 2007; 76: 013833.

50 Perina J Jr, Hamar M, Michálek V, Haderka O. Photon-number distributions of twin beams generated in spontaneous parametric down-conversion and measured by an intensified CCD camera. Phys Rev A 2012; 85: 023816.

51 Iskhakov TS, Usenko VC, Andersen UL, Filip R, Chekhova MV et al. Heralded source of bright multi-mode mesoscopic sub-Poissonian light. Opt Lett 2016; 41: 2149-2152.

52 Iskhakov TS, Chekhova MV, Leuchs G. Generation and direct detection of broadband mesoscopic polarization-squeezed vacuum. Phys Rev Lett 2009; 102: 183602.

53 Tapster PR, Seward SF, Rarity JG. Sub-shot-noise measurement of modulated absorption using parametric down-conversion. Phys Rev A 1991; 44: 3266-3269.

54 Whittaker R, Erven C, Neville A, Berry M, O'Brien JL et al. Absorption spectroscopy at the ultimate quantum limit from single-photon states. New J Phys 2017; 19: 023013.

55 Simon DS, Jaeger G, Sergienko AV. Quantum information in communication and imaging. Int J Quantum Inform 2014; 12: 1430004.

(c) (i) $\ominus$ This work is licensed under a Creative Commons AttributionBY NC ND NonCommercial-NoDerivs 4.0 International License. The images or other third party material in this article are included in the article's Creative Commons license, unless indicated otherwise in the credit line; if the material is not included under the Creative Commons license, users will need to obtain permission from the license holder to reproduce the material. To view a copy of this license, visit http:// creativecommons.org/licenses/by-nc-nd/4.0/

(C) The Author(s) 2017

Supplementary Information for this article can be found on the Light: Science \& Applications' website (http://www.nature.com/lsa). 